Relations industrielles

Industrial Relations

\title{
Managerial Process and Organizational Behavior, by Alan C. Filley \& Robert J. House-Scott, Foresman and Company; Glenview, Illinois, 1969, 500 pp.
}

\section{Jean Ladouceur}

\section{Volume 24, numéro 4, 1969}

URI : https://id.erudit.org/iderudit/028088ar

DOI : https://doi.org/10.7202/028088ar

Aller au sommaire du numéro

Éditeur(s)

Département des relations industrielles de l'Université Laval

ISSN

0034-379X (imprimé)

1703-8138 (numérique)

Découvrir la revue

Citer ce compte rendu

Ladouceur, J. (1969). Compte rendu de [Managerial Process and Organizational Behavior, by Alan C. Filley \& Robert J. House-Scott, Foresman and Company; Glenview, Illinois, 1969, 500 pp.] Relations industrielles / Industrial Relations, 24(4), 813-814. https://doi.org/10.7202/028088ar

Tous droits réservés (C) Département des relations industrielles de l'Université Laval, 1969
Ce document est protégé par la loi sur le droit d'auteur. L’utilisation des services d’Érudit (y compris la reproduction) est assujettie à sa politique d'utilisation que vous pouvez consulter en ligne.

https://apropos.erudit.org/fr/usagers/politique-dutilisation/ 
tementaux, conflits syndicats-direction. On y décrit les mécanismes de solution des conflits; celui de la théorie des jeux en particulier.

Avec le chapitre XIII, on retourne au domaine des applications en discutant des principes, des méthodes et de l'efficacité des programmes de formation pour dirigeants. En conclusion, l'auteur traite de l'entreprise de l'avenir et fait largement usage des thèses de Galbraith, de Likert, de Stalker et Burns.

En touchant ainsi une foule de thèmes, un auteur peut difficilement échapper au traitement superficiel d'un sujet. Pour parer à cette difficulté et pour apporter plus d'information, l'auteur insère dans un rectangle un résumé d'articles intéressants publiés dans des revues spécialisées.

L'auteur se proposait de donner une vue 《compréhensive» de la masse d'information dont on dispose actuellement sur les déterminants structurels et personnels du comportement organisationnel. Il voulait aussi mettre de l'ordre dans cet amas de connaissances. Nous croyons que l'auteur a atteint son premier objectif mais non le second.

\section{Laurent BELANGER}

Emerging Concepts in Management, by Max Wortman and Fred Luthans, Macmillan, New York, 1969, 462 pp.

Ce livre contient une sélection de 46 articles publiés depuis moins de cinq années. Ces articles sont représentatifs des récents développements dans la littérature administrative.

La première partie analyse les fondements théoriques et sociaux de l'administration. On y rappelle les difficultés d'unifier les multiples philosophies et théories publiées jusqu'à ce jour. La seconde partie analyse les fonctions administratives: planification, organisation, direction, contrôle. La contribution des sciences sociales est le sujet de la troisième partie; on $y$ traite des recherches récentes en comportement et changement organisationnel, en motivation et intégration personnelle.

Les sections IV et $\mathrm{V}$ de l'ouvrage sont consacrées à l'approche quantitative (re- cherche opérationnelle) et à l'analyse systématique en prenant l'organisation comme cadre de référence. $\mathrm{La}$ dernière partie présente des perspectives nouvelles sur l'administration multinationale et l'avenir des organisations.

Il convient d'indiquer quelques particularités. Chaque section est précédée d'une introduction et est suivie de questions à discuter et de lectures supplémentaires recommandées. Les articles ont été sélectionnés pour donner une vue d'ensemble des théories administratives et des tendances futures. Leurs auteurs appartiennent à plusieurs Ecoles de pensée, de sorte que le lecteur est stimulé à faire un jugement personnel sur les opinions exprimées.

Cet ouvrage est recommandé à quiconque veut mettre à jour ses connaissances sur les théories et concepts administratifs.

Jean LADOUCEUR

\section{Managerial Process and Organizational} Behavior, by Alan C. Filley \& Robert J. House,-Scott, Foresman and Company ; Glenview, Illinois, 1969, $500 \mathrm{pp}$.

This excellent book integrates recent theories in social sciences and classical management theory. The objective of the authors was to bring together and make operational the relevant findings from research in the litterature of sociology, psychology, political science, economics and business management. The topics are presented in an original way, much different from classical textbooks.

The book is devided into three parts. The first presents information on management thought, the fundamentals of research strategy, and authority structures. The second part deals with particular issues in organization theory : division of labor, organizational and personal objectives, planning, decisionmaking, role conflict, etc. The framework used is «theory - propositions evaluation »; the theory surrounding each issue is explained and resumed in key propositions, then evaluated by research findings. The third part treats leadership, motivation and organizational development. Chapter 18 gives a new 
model of organizational growth, with a different approach than those of Schroeder, Haire, Dale and Buchele.

Original presentation, good methodology, extensive bibliography, some innovation, all this makes the Filley-House book an excellent text for anyone interested in recent developments in administrative and behavioral sciences.

\section{J. LADOUCEUR}

Lexique de la psychologie, par Arlette et Roger MUCCHIELLI, Entreprise Moderne d'édition, Editions Sociales Françaises, Paris, 1969, 188 pp.

Lexique des sciences sociales, par Arlette et Roger MUCCHIELLI, Entreprise Moderne d'Edition, Editions Sociales Françaises, Paris, 1969, 196 pp.

Avec le vocabulaire technique que doivent nécessairement utiliser ceux qui veulent faire un travail scientifique, il devient de plus en plus difficile aux non-spécialistes de comprendre les études présentées au public sous forme d'articles ou d'ouvrages. Le domaine des sciences de l'homme est particulièrement peuplé de mots nouveaux ou de mots anciens pris dans une acception particulière.

Le Lexique de la psychologie contient les principaux concepts de la psychophysiologie, de la psychopathologie, de la psychanalyse, des psychothérapies, de la psychopédagogie et de la psychologie des groupes. Le Lexique des sciences sociales est consacré à la psychologie sociale, à la dynamique des groupes, à l'ergonomie, à la sociologie du travail et aux théories des communications et de l'information.

Ces deux ouvrages viennent aider les étudiants, les profanes et même les praticiens. Ils sont loin d'être complets et on pourra discuter l'une ou l'autre des définitions ou des explications que les auteurs proposent. II n'en reste pas moins que sous une présentation simple et d'une lecture facile, ils sont très utiles.
Les cadres et la révolution informatique, l'adaptation nécessaire, par Arnold Kaufmann, Entreprise moderne d'édition, Paris, 1968, 144 pages.

Arnold Kaufmann nous présente ici un sujet fleuve. Son intention est de motiver le lecteur et de l'amener à réfléchir sur la nécessaire éducation permanente à l'âge de l'ordinateur. Il nous présente ce qu'il appelle un combat contre certains préjugés et contre de nombreuses habitudes assez anciennes. S'adressant au cadre, il veut le persuader que son rôle dans la nouvelle société, sera justement de créer, encore plus que les autres, de manière que ces autres aient envie de se surmonter à leur tour. Ses considérations qui s'étendent sur un peu plus d'une centaine de pages, sont recoupées en quelque vingt-six chapitres.

L'auteur traite évidemment des cadres, surtout des cadres français. A partir d'études psycho-sociologiques, il en donne les caractéristiques principales et décrit particulièrement leurs attitudes face à l'ère des ordinateurs. La technologie de l'ordinateur et l'adaptation nécessaire de l'homme à ce nouvel instrument sont ensuite les préoccupations qui donnent lieu au plus grand nombre de chapitres. L'adaptation en termes de connaissances se traduit, entre autres, par le chapitre 8: «De nouvelles et indispensables connaissances pour les cadres $»$. L'importance des mathématiques modernes, la spécialisation et la qualification adaptable, l'imprégnation culturelle de même que l'intensification du dialogue homme-homme sont les sujets de chapitres subséquents. Des considérations plus globales sur la place de l'homme dans cette nouvelle société se traduisent par des chapitres comme: «Capitalisme et socialisme à l'ère des ordinateurs», «Les banques de données ou la liberté trahie », «La civilisation promotionnelle ». M. Kaufmann termine par quelques remarques supplémentaires sur le rôle des cadres et leur éducation permanente.

Cet ouvrage peut reconcilier avec l'informatique les cadres qui seraient peu ou pas convaincus. Nous souhaitons, avec l'auteur, qu'il puisse atteindre son but. 